\title{
Development of 23 novel microsatellite markers of Amomum tsao-ko (Zingiberaceae) based on restriction-site-associated DNA sequencing
}

\author{
Bingyue $\mathrm{Lu}^{1}{ }^{1} \cdot$ Mengli Ma ${ }^{1} \cdot$ Wei Zhang ${ }^{1} \cdot$ Hengling Meng ${ }^{1} \cdot$ En Lei $^{1} \cdot$ Tiantao Wang $^{1} \cdot$ Chunyan $\mathrm{Li}^{1}$
}

Received: 10 June 2020 / Accepted: 24 December 2020 / Published online: 17 January 2021

(c) The Author(s), under exclusive licence to Springer Nature B.V. part of Springer Nature 2021

\begin{abstract}
Amomum tsao-ko (Zingiberaceae) is a traditional Chinese medicine and condiment, and an important economic crop in the tropical forest of southwest China. However, few simple sequence repeat (SSR) markers are available in A. tsao-ko, which is hindering genetic research in this species. The aim of this study was to develop and characterize microsatellite markers for A. tsao-ko using restriction-site-associated DNA sequencing. A total of 115,482 microsatellites were identified using MISA software, and 13,411 SSR primer pairs were designed. 100 pairs of SSR primers were selected at random and used to evaluate polymorphisms among 4 A. tsao-ko samples. Finally, 23 pairs of SSR primers with clear bands and obvious polymorphism were selected for genetic diversity analysis of $72 \mathrm{~A}$. tsao-ko accessions. The number of alleles and effective number of alleles per locus ranged from 2 to 6 and from 1.315 to 3.776, respectively. The observed heterozygosity ranged from 0.208 to 0.779 , and the expected heterozygosity was from 0.239 to 0.735 . The average values of the polymorphic information content were 0.454 . Hardy-Weinberg equilibrium (HWE) analysis showed that 10 loci significantly deviated from HWE $(P<0.05)$. The pairwise $\mathrm{F}_{\mathrm{ST}}$ and genetic distance values revealed low levels of genetic differentiation and high genetic similarity among six A. tsao-ko populations. These microsatellite markers developed will provide a valuable tool for further germplasm characterization, genetic diversity, and breeding studies in A. tsao-ko.
\end{abstract}

Keywords Amomum tsao-ko $\cdot$ Microsatellite $\cdot$ Genetic diversity $\cdot$ Zingiberaceae

\section{Introduction}

Amomum tsao-ko, commonly known as black cardamom, is a perennial evergreen tufted herb belonging to the family Zingiberaceae. It is mainly distributed in Southwest China and Northern Vietnam, and found at altitudes ranging from 1300 to $1800 \mathrm{~m}$. It is a type of economical crop that can grow in humid forests in tropical and subtropical areas [1, 2]. Yunnan province is the main A. tsao-ko-producing area in China, contributing to $95 \%$ of the nation's output [3]. The whole plant of A. tsao-ko has a spicy smell, is edible and

Supplementary Information The online version contains supplementary material available at https://doi.org/10.1007/s1103 3-020-06127-6.

Bingyue Lu 1by202@126.com

$1 \quad$ Key Laboratory for Research and Utilization of Characteristic Biological Resources in Southern Yunnan, College of Life Science and Technology, Honghe University, Mengzi, Yunnan, People's Republic of China used medicinally. Its dried fruit is often used to treat internal resistance to cold and dampness, epigastric distension, fullness and vomiting, and malaria among other ailments [4, 5]. Recently, A. tsao-ko was one of the clinical prescriptions for patients with light, ordinary and severe cases of COVID19 in China, demonstrating its important role in antiviral therapy $[6,7]$. In food, A. tsao-ko has become a top-grade seasoning, and widely used in food processing [8].

Crop breeding effectiveness not only depends on the number of germplasm resources but also largely depends on the mastery of genetic characteristics of the diversity of these germplasm resources. Previous studies on A. tsao-ko have primarily concentrated on chemical composition analysis and pharmacological action, and there are few reports on genetic diversity [9-11]. Microsatellite markers, also called simple sequence repeat (SSR) markers, are effective in estimating genetic diversity and relationships within and among populations, and are widely used in plant genetic research. Yang et al. [12] developed 24 pairs of SSR primers by FIASCO technique for $A$. tsao-ko, but only nine of them were polymorphic, such limited SSR markers are not enough 
to conduct comprehensive genetic studies. The objectives of this study were to develop a set of novel microsatellite markers using restriction-site-associated DNA sequencing (RAD-seq) technique. These markers will provide a useful tool for A. tsao-ko genetic research.

\section{Materials and methods}

A total of 72 accessions from six cultivated populations of $A$. tsao-ko were collected from Yunnan Province, China (Fig. 1, Table S1). The collections covered the main producing area, with the longitudes ranging from $99^{\circ} 49^{\prime} 12^{\prime \prime}$ (LC, Lancang population) to $103^{\circ} 31^{\prime} 48^{\prime \prime}$ (PB, Pingbian population), latitudes ranging from $22^{\circ} 53^{\prime} 24^{\prime \prime}$ (LVC, Lvchun population) to $24^{\circ} 17^{\prime} 24^{\prime \prime}$ (YX, Yunxian population), and elevations spanning from 1665 to $2108 \mathrm{~m}$. Each population consisted of 12 individuals, and the linear distance between individuals in the same population is not less than $50 \mathrm{~m}$ to decrease the possibility of sampling from the same clone.

The genomic DNA of $A$. tsao-ko was extracted using a cetyltrimethylamine bromide method [13], and the concentration and quality of DNA were detected by NanoDrop 2000 ultra-micro spectrophotometer. The working solutions of genomic DNA (20 ng/ $\mu \mathrm{L}$ ) of different samples were stored at $4{ }^{\circ} \mathrm{C}$. Six genomic DNA samples (one sample from each population) of A. tsao-ko were mixed with the same amount, and the RAD-seq technique was used to simplify sequencing. The bidirectional sequencing of 350-650 bp recovery sequence was carried out by HiSeq 4000 sequencing platform with PE150 mode. To obtain clean reads, the adapters and sequences $<50 \mathrm{bp}$ and low-quality reads were removed. The clean reads were assembled using SOAPdenovo2.04 [14]. The sequencing data were deposited in the Sequence Read Archive (SRA) of NCBI database (SRA Accession: PRJNA529582).

The software MISA was used to search the SSR sites of the DNA sequences obtained by sequencing [15]. The configuration parameters are as follows: the minimum repetition times of single nucleotide, dinucleotide, trinucleotide, tetranucleotide, pentanucleotide and hexanucleotide were $10,5,4,4,4$ and 4 , respectively, and the sequence length between two adjacent SSR is $\geq 12$ bp. SSR primers were designed based on the flanking sequence of SSR locus using Primer 3.0 software [16]. The parameters were set as follows: GC content 40-60\%, primer length 18-27 bp, annealing temperature $50-65^{\circ} \mathrm{C}$, and amplification product length 100-300 bp.

100 pairs of SSR primers were randomly selected for synthesis and were screened by four A. tsao-ko samples. Finally, 23 pairs of SSR primers with clear bands and obvious polymorphism were selected for genetic diversity analysis of 72 A. tsao-ko accessions. The $10 \mu \mathrm{L}$ polymerase chain reaction (PCR) system consists of $1.0 \mu \mathrm{L}$ template DNA $(20 \mathrm{ng} / \mu \mathrm{L})$,
Fig. 1 Map of A. tsao-ko samples collection sites. PB, Pingbian population; JP, Jinping population; YY, Yuanyang population; LVC, Lvchun population; YX, Yunxian population; LC, Lancang population (see Table S1 for full description of the populations)

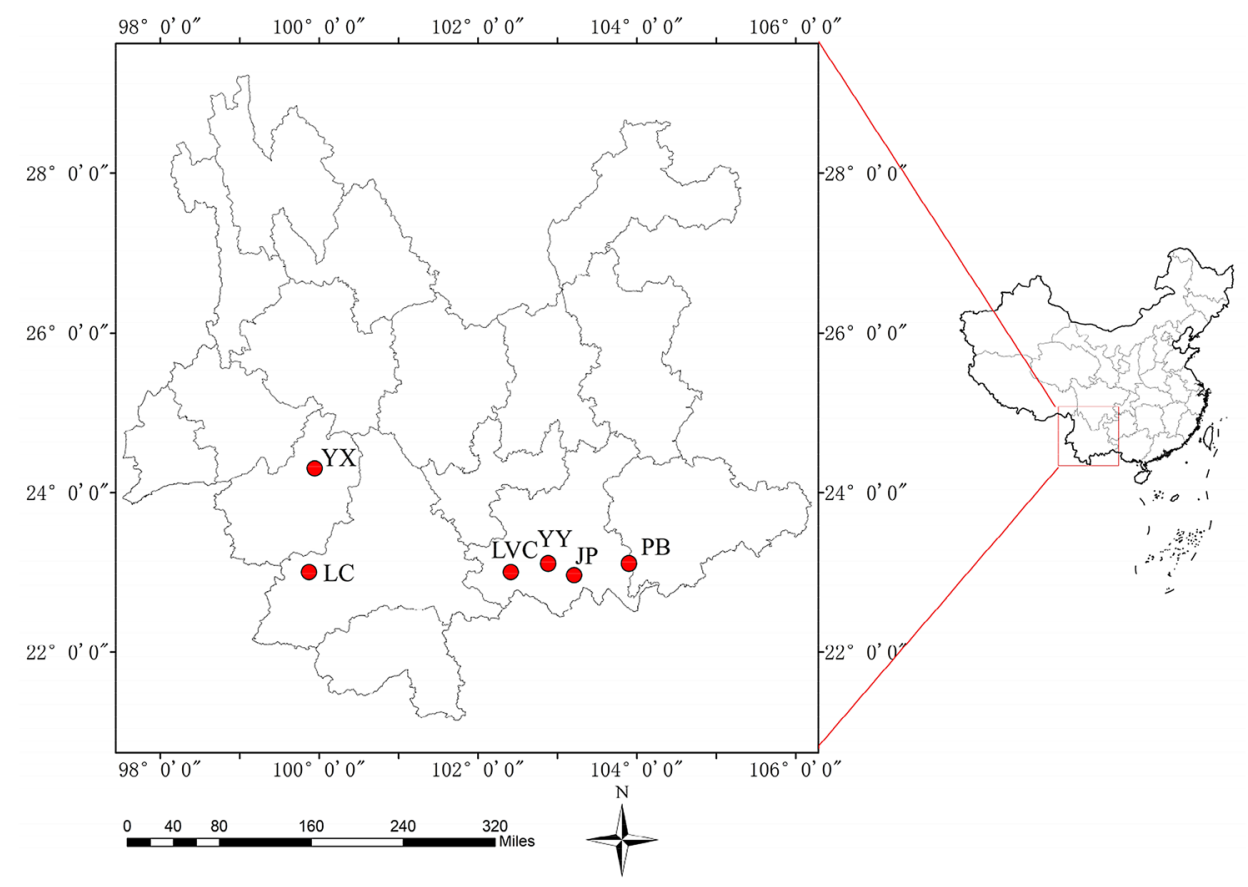


$0.8 \mu \mathrm{L} \mathrm{SSR} \mathrm{primers}(0.2 \mu \mathrm{mol} / \mathrm{L}), 0.8 \mu \mathrm{L}$ deoxyribonucleoside triphosphates (dNTPs) $(2.5 \mathrm{~mol} / \mathrm{L}), 1.2 \mu \mathrm{L} 10 \times \mathrm{PCR}$ buffer (including $\mathrm{Mg}^{2+}$ ), $0.2 \mu \mathrm{L}$ Taq DNA polymerase $(2.5 \mathrm{u} / \mu \mathrm{L})$, and $6 \mu \mathrm{L} \mathrm{ddH}_{2} \mathrm{O}$. The PCR procedure included pre-denaturation $\left(95{ }^{\circ} \mathrm{C} / 5 \mathrm{~min}\right)$, followed by $95{ }^{\circ} \mathrm{C} / 30 \mathrm{~s}$, $51-55^{\circ} \mathrm{C} / 30 \mathrm{~s}, 72{ }^{\circ} \mathrm{C} / 30 \mathrm{~s}$, a total of 30 cycles, and finally extended at $72{ }^{\circ} \mathrm{C}$ for $10 \mathrm{~min}$ to preserve at $4{ }^{\circ} \mathrm{C}$. All of the PCR amplifications were conducted using a thermal cycler (ETC-811, Eastwin). The amplified products were separated on $8 \%$ non-denaturing polyacrylamide gel (acrylamide:bisac rylamide $=19: 1$, stained with $1 \%$ silver nitrate at the end of electrophoresis and photographed after the bands were clear.

For each locus, the evaluation parameters including the observed number of alleles (Na), effective number of alleles $(\mathrm{Ne})$, observed heterozygosity (Ho), expected heterozygosity $(\mathrm{He})$, fixation index $(\mathrm{F})$, genetic distance, and pairwise $\mathrm{F}_{\mathrm{ST}}$ values were calculated by GenAlEx 6.501 program [17], which are also used for the Hardy-Weinberg equilibrium (HWE) test. Frequencies of null alleles were estimated using Micro-checker 2.2.3 [18]. The polymorphism information content (PIC) of polymorphic loci was calculated using PowerMarker V3.25 [19]. 72 accessions were assessed by multivariate principal coordinate analysis $(\mathrm{PCoA})$ performed with GenAlEx 6.501. A Mantel test was performed to determine the correlation between genetic distance and geographic distance matrices using NTSYS-pc 2.11 software [20].

\section{Results and discussion}

By RAD-seq, 5,269,456 (1,317,364,000 bp) raw reads were obtained, and 5,117,655 (1,185,485,454 bp) clean reads were obtained after filtering. A total of 115,482 microsatellites were identified using MISA software, and 13,411 SSR primer pairs were designed using Primer 3.0. 100 pairs of randomly selected primers were validated in four A. tsao$k o$ accessions, of which 95 pairs could amplify bands in the target area, 64 pairs were polymorphic (Table S2). 23 primer pairs with clear bands and obvious polymorphism were selected for further analysis (Table 1).

A total of 90 bands were amplified by 23 SSR markers in $72 \mathrm{~A}$. tsao-ko, with an average of 3.913 bands per primer pairs. The total number of alleles (Na) per loci varied from 2 (AM65) to 6 (AM49 and AM89), and the effective number of alleles (Ne) ranged from 1.315 (AM43) to 3.776 (AM89). The average $\mathrm{Ne}$ (2.305) was lower than the $\mathrm{Na}$ (3.913) thus reflecting imbalanced allelic frequencies in populations, which could imply the presence of rare or unique alleles.
The observed heterozygosity (Ho) and expected heterozygosity (He) in each locus vary from 0.208 (AM43) to 0.779 (AM42) and from 0.239 (AM43) to 0.735 (AM89), respectively. All the genetic parameters were higher than those of SSR markers developed by Yang et al [12]. The PIC ranged from 0.228 (AM43) to 0.709 (AM89). The mean fixation index was 0.072 , and values ranged from -0.181 (AM42) to 0.272 (AM13), indicating that there was no inbreeding depression in A. tsao-ko population. Among all the loci, 5 loci (AM4, AM12, AM13, AM40, and AM44) were exhibited heterozygote deficiency, as indicated by the positive and significant fixation index. Heterozygote deficiency could be the result of rare alleles, correlations among loci, or the sampling of closely related individuals. HWE analysis showed that 10 loci (AM4, AM6, AM8, AM12, AM13, AM42, AM43, AM44, AM45, and AM49) significantly deviated from HWE $(P<0.05)$, and the remaining 13 loci (AM1, AM7, AM9, AM17, AM27, AM29, AM36, AM40, AM41, AM65, AM89, AM94, and AM99) were in accordance with HWE (Table 2). Micro-checker analysis showed that null alleles frequencies were lower than 0.2 for each locus, indicating these HWE deviations may be caused by anthropic selection and short-cloning history in cultivated rather than null alleles [12, 21].

In this study, PCoA analysis did not clearly separate the populations, and the germplasm from different populations were mixed with each other (Fig. 2). Consistent to this result, the Mantel test revealed that there was no significant correlation between geographic and genetic distance $(r=0.076$, $p=0.579$ ), which suggested no significant geographic restriction to gene flow among the populations. The pairwise $\mathrm{F}_{\mathrm{ST}}$ values ranged from 0.029 ( $\mathrm{LVC}$ and $\mathrm{LC}$ ) to 0.074 (YY and LC), with an average of 0.047. The genetic distance varied from 0.056 (JP and YX) to 0.146 (YY and LC) with an average of 0.072 (Table 3). These results showed a relatively low degree of genetic differentiation and high genetic similarity among these populations. A. tsao-ko is a small group of species that is geographically restricted to southern China (Yunnan), northern Laos and Vietnam at altitudes ranging from 1300 to $1800 \mathrm{~m}$, where it thrives in well-drained, moist, organic-matter-rich soil in shade or partial shade $[4,22]$. Narrow distribution areas and special habitats lead to the high genetic similarity between $A$. tsao-ko populations, similar results were also observed in Dendrocalamus membranaceus [23], Thuja sutchuenensis [24] and Stemona parviflora [25]. Furthermore, a high level of gene flow $(\mathrm{Nm}=5.425)$ was detected among A. tsao-ko populations based on random amplified polymorphic DNA 
Table 1 Characterization of 23 microsatellite loci developed for A. tsao-ko
Locus Primer sequences $\left(5^{\prime}-3^{\prime}\right)$

Repeat motif $\quad \mathrm{Ta}\left({ }^{\circ} \mathrm{C}\right) \quad$ Size (bp) $\quad$ GenBank accession number

\begin{tabular}{|c|c|c|c|c|c|}
\hline \multirow[t]{2}{*}{ AM1 } & F: CGCAGAATTAACCACCAGCG & (A) 16 & 55 & 135 & MT495611 \\
\hline & R: TGCCTCTCAATTGTTCAGGACA & & & & \\
\hline \multirow[t]{2}{*}{ AM4 } & F: AGGGATAGAAGACAGCCAGGA & (AAAC) 5 & 55 & 150 & MT525020 \\
\hline & R: TGAGGTTTCTTCCGCACTGT & & & & \\
\hline \multirow[t]{2}{*}{ AM6 } & F: GCCAAGAGCATATTCAGTGAGT & $(\mathrm{AAC}) 7$ & 53 & 137 & MT525021 \\
\hline & R: ATAACCGACCCCACCTAGTG & & & & \\
\hline \multirow[t]{2}{*}{ AM7 } & F: CGCGCTTGTTTGGTTGGTTA & (AAT) 10 & 55 & 146 & MT525022 \\
\hline & R: TCCCTTGCTTGCCTGCTATT & & & & \\
\hline \multirow[t]{2}{*}{ AM8 } & F: CGAACAAACATGCCCGTGAA & $(\mathrm{AAT}) 8$ & 55 & 141 & MT525023 \\
\hline & R: CGACAAGGTAACGGGTTCCA & & & & \\
\hline \multirow[t]{2}{*}{ AM9 } & F: TGGGAGATCGAGGAAGGAAGA & (AATT) 5 & 55 & 135 & MT525024 \\
\hline & R: GCCTGCACCGCCATTTTTAT & & & & \\
\hline \multirow[t]{2}{*}{ AM12 } & F: AGCAAGTGACACTCTCATCT & $(\mathrm{AG}) 13$ & 51 & 133 & MT525025 \\
\hline & R: ACTTGAGAGATACACAATGTGTT & & & & \\
\hline \multirow[t]{2}{*}{ AM13 } & F: GACGTAGCTGCACTGCAATG & (AG) 8 & 55 & 144 & MT525026 \\
\hline & R: CCGAACAAACCAACCGAACC & & & & \\
\hline \multirow[t]{2}{*}{ AM17 } & F: TGTGGTAATCAAACGCGACG & (AGAGGG)5 & 54 & 137 & MT525027 \\
\hline & R: CGTCGAAGTCTGTCATATCTGC & & & & \\
\hline \multirow[t]{2}{*}{ AM27 } & F: GAGGGAAGCGAATCTCCGAG & $($ ATCGAA) 5 & 55 & 151 & MT525028 \\
\hline & R: GTCCTCTCCTTCTCGGCATC & & & & \\
\hline \multirow[t]{2}{*}{ AM29 } & F: ACGTACCAGTACAAACCTCAGA & $(\mathrm{ATT}) 8$ & 54 & 143 & MT525029 \\
\hline & R: ACGTGTTAGCGACGAAATTAGT & & & & \\
\hline \multirow[t]{2}{*}{ AM36 } & F: ACAAGAATAAATGCCCCGAA & (CTATTC) 5 & 53 & 176 & MT525030 \\
\hline & R: TGTTTGTAGTGTTCGCGTGC & & & & \\
\hline \multirow[t]{2}{*}{ AM40 } & F: TGGTACCACAAAACTCTGCCA & $(\mathrm{CCT}) 6$ & 55 & 154 & MT525031 \\
\hline & R: TGCCTGGCCAGTAAATTAAGGA & & & & \\
\hline \multirow[t]{2}{*}{ AM41 } & F: GGCCTCAAGGTGTTCGTGTA & $(\mathrm{CCT}) 8$ & 55 & 143 & MT525032 \\
\hline & R: AGGAGCTCCTCAACATCATCG & & & & \\
\hline \multirow[t]{2}{*}{ AM42 } & F: GTAATTAGCAGGCACGACGC & (CCTCTG) 5 & 55 & 149 & MT525033 \\
\hline & R: ATCGTTGAAGTGTGGACGCT & & & & \\
\hline \multirow[t]{2}{*}{ AM43 } & F: ACGACTAACCGATCGCCTTC & $(\mathrm{CGA}) 8$ & 55 & 137 & MT525034 \\
\hline & R: CGTTCTCATCTCCGGAGTCG & & & & \\
\hline \multirow[t]{2}{*}{ AM44 } & F: CTTCCGACAAAGGGCTGAGT & (CGC) 11 & 55 & 133 & MT525035 \\
\hline & R: CCACGATGGAGTTAAGGCTGA & & & & \\
\hline \multirow[t]{2}{*}{ AM45 } & F: GAGCAAGGCAGCAGATTTCG & $(\mathrm{CGC}) 6$ & 55 & 143 & MT525036 \\
\hline & R: CGCTTCTCCGGAGTCGTAAA & & & & \\
\hline \multirow[t]{2}{*}{ AM49 } & F: TCGGCTTGGAGGTAGCTAGA & (CT) 11 & 55 & 151 & MT525037 \\
\hline & R: AGGTAGGTAGGTGGTGCTGT & & & & \\
\hline \multirow[t]{2}{*}{ AM65 } & F: TTCCCCGACAAAACTAGGGC & $(\mathrm{GCA}) 6$ & 55 & 149 & MT525038 \\
\hline & R: AGCAACGAGATCAAGGGCAA & & & & \\
\hline \multirow[t]{2}{*}{ AM89 } & F: GTCGTCGATTGACTCCGTCA & $(\mathrm{TCT}) 7$ & 55 & 157 & MT525039 \\
\hline & R: TCCATGTTCTGCTGCGAAGT & & & & \\
\hline \multirow[t]{2}{*}{ AM94 } & F: GATGTGCGGCGAGACAGATA & (TTAA) 5 & 55 & 146 & MT525040 \\
\hline & R: GGCTGAGCCTGAGAGTACAG & & & & \\
\hline \multirow[t]{2}{*}{ AM99 } & F: AACCACTCGAACGACCCTTC & (TTGCCG)5 & 55 & 138 & MT525041 \\
\hline & R: CAACGTCCAAAAGCAGAAGCA & & & & \\
\hline
\end{tabular}


Table 2 Genetic variation of the 23 polymorphic microsatellite loci in 72 accessions of $A$. tsao-ko

\begin{tabular}{|c|c|c|c|c|c|c|c|c|}
\hline Locus & $\mathrm{Na}$ & $\mathrm{Ne}$ & Ho & $\mathrm{He}$ & PIC & $\mathrm{F}$ & FNA & HWE \\
\hline AM1 & 4 & 2.444 & 0.514 & 0.591 & 0.516 & 0.130 & 0.073 & $0.233^{\mathrm{ns}}$ \\
\hline AM4 & 3 & 2.461 & 0.479 & 0.594 & 0.533 & $0.193^{*}$ & 0.129 & $0.046^{*}$ \\
\hline AM6 & 3 & 2.056 & 0.514 & 0.514 & 0.395 & -0.001 & 0.013 & $0.000 * * *$ \\
\hline AM7 & 4 & 1.877 & 0.472 & 0.467 & 0.413 & -0.011 & 0.039 & $0.961^{\mathrm{ns}}$ \\
\hline AM8 & 3 & 1.650 & 0.366 & 0.394 & 0.359 & 0.070 & -0.022 & $0.006 * *$ \\
\hline AM9 & 5 & 2.555 & 0.571 & 0.609 & 0.580 & 0.061 & 0.038 & $0.972^{\mathrm{ns}}$ \\
\hline AM12 & 4 & 1.403 & 0.211 & 0.287 & 0.302 & $0.265^{*}$ & 0.139 & $0.000 * * *$ \\
\hline AM13 & 3 & 2.062 & 0.375 & 0.515 & 0.454 & $0.272^{*}$ & -0.033 & $0.000 * * *$ \\
\hline AM17 & 4 & 1.864 & 0.493 & 0.463 & 0.399 & -0.064 & 0.154 & $0.202^{\mathrm{ns}}$ \\
\hline AM27 & 3 & 2.223 & 0.606 & 0.550 & 0.479 & -0.101 & -0.051 & $0.401^{\mathrm{ns}}$ \\
\hline AM29 & 4 & 1.704 & 0.394 & 0.413 & 0.387 & 0.045 & 0.041 & $0.860^{\mathrm{ns}}$ \\
\hline AM36 & 4 & 1.706 & 0.431 & 0.414 & 0.387 & -0.040 & 0.124 & $0.220^{\mathrm{ns}}$ \\
\hline AM40 & 4 & 2.741 & 0.486 & 0.635 & 0.571 & $0.235^{*}$ & 0.060 & $0.153^{\mathrm{ns}}$ \\
\hline AM41 & 5 & 1.810 & 0.403 & 0.447 & 0.415 & 0.100 & -0.096 & $0.916^{\mathrm{ns}}$ \\
\hline AM42 & 4 & 2.939 & 0.779 & 0.660 & 0.655 & -0.181 & 0.080 & $0.000 * * *$ \\
\hline AM43 & 4 & 1.315 & 0.208 & 0.239 & 0.228 & 0.129 & 0.107 & $0.010^{*}$ \\
\hline AM44 & 4 & 2.598 & 0.486 & 0.615 & 0.543 & $0.210^{*}$ & 0.008 & $0.001 * *$ \\
\hline AM45 & 5 & 2.377 & 0.556 & 0.579 & 0.515 & 0.041 & 0.079 & $0.001 * *$ \\
\hline AM49 & 6 & 1.942 & 0.414 & 0.485 & 0.460 & 0.146 & 0.068 & $0.000 * * *$ \\
\hline AM65 & 2 & 1.591 & 0.324 & 0.371 & 0.323 & 0.128 & -0.022 & $0.281^{\mathrm{ns}}$ \\
\hline AM89 & 6 & 3.776 & 0.771 & 0.735 & 0.709 & -0.049 & 0.058 & $0.673^{\text {ns }}$ \\
\hline AM94 & 3 & 1.584 & 0.333 & 0.369 & 0.328 & 0.096 & -0.009 & $0.342^{\mathrm{ns}}$ \\
\hline AM99 & 3 & 2.267 & 0.569 & 0.559 & 0.492 & -0.019 & -0.025 & $0.979^{\mathrm{ns}}$ \\
\hline Mean & 3.913 & 2.305 & 0.468 & 0.500 & 0.454 & 0.072 & & \\
\hline
\end{tabular}

$\mathrm{Na}$, number of alleles; Ne, effective number of alleles; Ho, observed heterozygosity; He, expected heterozygosity; PIC, polymorphism information content; F, fixation index, *significance value from $95 \%$ confidence interval; FNA, frequency of null alleles; HWE deviation from Hardy-Weinberg equilibrium (ns, no significant, $* \mathrm{p}<0.05, * * \mathrm{p}<0.01, * * * \mathrm{p}<0.001)$

\section{Principal Coordinates (PCoA)}

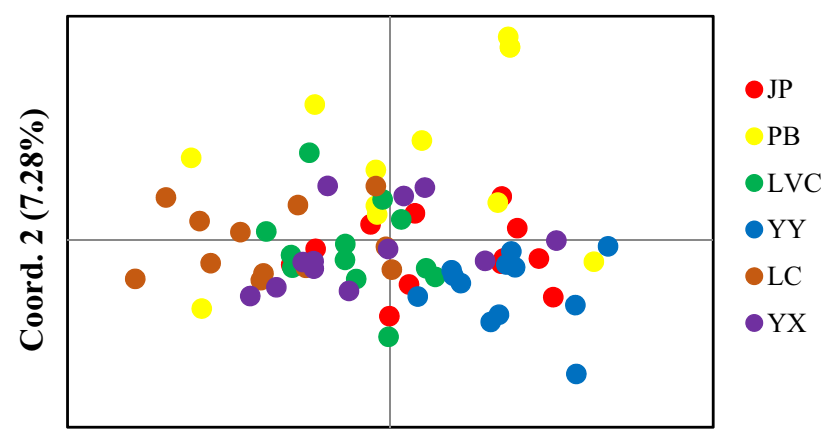

Coord. $1(7.84 \%)$
(RAPD) markers in our previous study [8]. Such gene flow was enough to resist the genetic differentiation caused by genetic drift. In conclusion, we developed and characterized 23 novel microsatellite markers in A. tsao-ko. The set of markers significantly increases the availability of polymorphic molecular markers for the species, and will provide a valuable resource for genetic diversity, core collection construction, genetic mapping and marker-assisted selection breeding in A. tsao-ko.

Fig. 2 Principal coordinate analysis based on the genotyping of 72 accessions of $A$. tsao-ko with 23 microsatellite loci 
Table 3 Pairwise of $\mathrm{F}_{\mathrm{ST}}$ (above diagonal) and genetic distance (below diagonal) for six $A$. tsao-ko populations

\begin{tabular}{lllllll}
\hline Population & JP & PB & LVC & YY & LC & YX \\
\hline JP & - & 0.052 & 0.034 & 0.034 & 0.052 & 0.032 \\
PB & 0.095 & - & 0.047 & 0.064 & 0.057 & 0.059 \\
LVC & 0.058 & 0.096 & - & 0.043 & 0.029 & 0.033 \\
YY & 0.059 & 0.141 & 0.081 & - & 0.074 & 0.056 \\
LC & 0.093 & 0.122 & 0.060 & 0.146 & - & 0.046 \\
YX & 0.056 & 0.112 & 0.057 & 0.111 & 0.084 & - \\
\hline
\end{tabular}

Acknowledgements This work was supported by National Natural Science Foundation of China (31460380), Yunnan Local Colleges Applied Basic Research Projects (202001BA070001-181), Youth Academic Backbone Project of Honghe University (2014GG0101) and Agricultural Discipline Construction Project of Honghe University (2018ZDXK02). We thank LetPub (www.letpub.com) for its linguistic assistance during the preparation of this manuscript.

Funding This study was funded by National Natural Science Foundation of China (31460380), Yunnan Local Colleges Applied Basic Research Projects (202001BA070001-181), Youth Academic Backbone Project of Honghe University (2014GG0101) and Agricultural Discipline Construction Project of Honghe University (2018ZDXK02).

\section{Compliance with ethical standards}

Conflict of interest The authors declare that they have no conflict of interest.

Ethical approval This article does not contain any studies with human participants or animals performed by any of the authors

\section{References}

1. National Pharmacopoeia Committee (2015) Pharmacopoeia of People's Republic of China. Chemical Industry Press, Beijing, $p$ 409

2. Liu GZ, Jin M, Cai CT, Ma CN, Chen ZZ, Gao LL (2019) Soil microbial community structure and physicochemical properties in Amomum tsaoko based agroforestry systems in the Gaoligong Mountains, Southwest China. Sustainability 11:546

3. Ma ML, Wang TT, Lei E, Meng HL, Zhang W, Zhang TT, Lu BY (2020) Genetic diversity analysis of Amomum tsao-ko in Jinping based on phenotypic traits and SSR markers. Crops 36:54-59 (in Chinese with English abstract)

4. Lim TK (2013) Edible medicinal and non-medicinal plants. Springer, Netherlands, pp 813-817

5. Shi YN, Jin H, Yang Y, Zhang LX, Zhu XY, Wang L, Ji PZ, Liu DH (2014) Herbal verification of medicinal Amomum tsao-ko. Med Plant 5:16-19, 24

6. Jin YH, Cai L, Cheng ZS et al (2020) A rapid advice guideline for the diagnosis and treatment of 2019 novel coronavirus (2019$\mathrm{nCoV}$ ) infected pneumonia (standard version). Mil Med Res 7:4

7. Yang Z, Ding ML, Jia KK, Ma ST, Ju WZ (2020) Exploring active compounds of Da-Yuan-Yin in treatment of COVID-19 based on network pharmacology and molecular docking method. Chin Tradit Herb Drugs 51:836-844 (in Chinese with English abstract)

8. Lu BY, Ma ML, Wang TT, Meng HL, Lei E, Zhang W (2018) Genetic diversity and genetic relationships of Amomum tsao-ko based on random amplified polymorphic DNA markers. Int J Agric Biol 20:2032-2038

9. Liu H, Yan QS, Zou DL, Bu XL, Zhang BJ, Ma XC, Leng AJ, Zhang HL, Li DW, Wang C (2018) Identification and bioactivity evaluation of ingredients from the fruits of Amomum tsaoko Crevost et Lemaire. Phytochem Lett 28:111-115

10. Kim JG, Jang H, Le TPL, Hong HR, Lee MK, Hong JT, Lee D, Hwang BY (2019) Pyranoflavanones and pyranochalcones from the fruits of Amomum tsao-ko. J Nat Prod 82:1886-1892

11. Sim S, Tan S, Kohlenberg B, Braun N (2019) Amomum tsaoko-Chinese black cardamom: detailed oil composition and comparison with two other cardamom species. Nat Prod Commun 14. https://doi.org/10.1177/1934578X19857675

12. Yang YW, Yang ZY, Yan MR, Qian ZG, Guan KY (2014) Isolation and characterization of microsatellite markers for Amomum tsaoko (Zingiberaceae), an economically important plant in China. Genet Mol Res 13:8220-8224

13. Doyle JJ (1990) Isolation of plant DNA from fresh tissue. Focus 12:13-15

14. Luo RB, Liu BH, Xie YL et al (2012) SOAPdenovo2: an empirically improved memory-efficient short-read de novo assembler. GigaScience 1:18

15. Thiel T, Michalek W, Varshney R, Graner A (2003) Exploiting EST databases for the development and characterization of genederived SSR-markers in barley (Hordeum vulgare L.). Theor Appl Genet 10:411-422

16. Untergasser A, Cutcutache I, Koressaar T, Ye J, Faircloth BC, Remm M, Rozen SG (2012) Primer 3-new capabilities and interfaces. Nucleic Acids Res 40:e115-e115

17. Peakall R, Smouse PE (2012) GenAlEx 6.5: genetic analysis in Excel. Population genetic software for teaching and research-an update. Bioinformatics 28:2537-2539

18. Van Oosterhout C, Hutchinson WF, Wills DPM, Shipley P (2004) Micro-checker: software for identifying and correcting genotyping errors in microsatellite data. Mol Ecol Notes 4:535-538

19. Liu K, Muse SV (2005) PowerMarker: an integrated analysis environment for genetic marker data. Bioinformatics 21:2128-2129

20. Rohlf FJ (2000) NTSYS-pc, numerical taxonomy and multivariate analysis system, version 2.1. Exceter software, New York

21. Shu WJ, Tang JM, Chen ZY, Jiang YS, Wang ZF, Wei X (2019) Analysis of genetic diversity and population structure in Sophora japonica Linn. in China with newly developed SSR markers. Plant Mol Biol Report 37:87-97

22. De Boer H, Newman M, Poulsen AD et al (2018) Convergent morphology in Alpinieae (Zingiberaceae): Recircumscribing Amomum as a monophyletic genus. Taxon 67:6-36

23. Yang HQ, An MY, Gu ZJ, Tian B (2012) Genetic diversity and differentiation of Dendrocalamus membranaceus (Poaceae: Bambusoideae), a declining bamboo species in Yunnan, China, as based on inter-simple sequence repeat (ISSR) analysis. Int J Mol Sci 13:4446-4457

24. Liu JF, Shi SQ, Chang EM, Yang WJ, Jiang ZP (2013) Genetic diversity of the critically endangered Thuja sutchuenensis revealed 
by ISSR markers and the implications for conservation. Int J Mol Sci 14:14860-14871

25. Zhang X, Ge J, Yang J, Dunn B, Chen G (2017) Genetic diversity of Stemona parviflora: A threatened myrmecochorous medicinal plant in China. Biochem Syst Ecol 71:193-199
Publisher's Note: Springer Nature remains neutral with regard to jurisdictional claims in published maps and institutional affiliations. 